\title{
Un teorema di esistenza per controlli ottimi di sistemi definiti da equazioni integrali di Urysohn $\left(^{*}\right)(* *)$.
}

\author{
Marta Luisa Bennatr (Genova)
}

Summary. - In this paper an existence theorem is proved for optimal control problems described by Urysohn systems, with pointwise constraints on controls and states. An example of application of the existence theorem is given.

\section{1. - Introduzione.}

Questo lavoro è dedicato allo studio di problemi di controllo ottimo per sistemi di equazioni di Urysohn. Il risultato ottenuto è un teorema di esistenza per problemi di ottimizzazione del tipo di Lagrange. Il teorema dimostrato generalizza risultati noti sull'argomento (v. [1] e [2]).

In [1] sono provati teoremi di esistenza per sistemi di controllo del tipo di Urysohn in eui l'equazione di stato è in forma meno generale di quella considerata nel presente lavoro e così in [2] dove il sistema di controllo è descritto da un'equazione integrale nonlineare di Volterra. Il risultato provato è un caso concreto rispetto a [3], ove l'equazione di stato è un'equazione integrale astratta. È dato un esempio di applicazione del teorema di esistenza.

\section{2. - Notazioni.}

Sia $\Omega$ un sottoinsieme (non ruoto), misurabile secondo Lebesgue e di misura finita dello spazio $\boldsymbol{R}^{r}$, con $r \geqslant 1$. La variabile stato $x$ è un elemento di $\boldsymbol{R}^{n}$; il vettore controllo $u$ è in $\boldsymbol{R}^{m}$.

Per ogni $t$ in $Q$ sia $A(t)$ un sottoinsieme non vuoto, chiuso dello spazio $\boldsymbol{R}^{n}$ e sia $A$ l'insieme di tutte le coppie $(t, x)$ con $t \in \Omega, x \in A(t)$. Per ogni $(t, x) \in A$, sia $U(t, x)$ un sottoinsieme non vioto dello spazio $\boldsymbol{R}^{m}$. Sia $M$ l'insieme degli elementi $(t, x, u)$ con $(t, x) \in A$ e $u \in U(t, x)$. Sono date le funzioni $h, g$ definite $s u \Omega^{2} \times \boldsymbol{R}^{n}$ a valori rispettivamente in $\boldsymbol{R}^{n}$ e matrici $n \times n ; f, f_{0}$ definite su $\Omega \times \boldsymbol{R}^{n} \times \boldsymbol{R}^{m}$ a valori in $\boldsymbol{R}^{n}$ e $\boldsymbol{R}$ rispettivamente. Sia $\varphi$ definita da $\Omega$ in $\boldsymbol{R}^{n}$.

(*) Entrata in Redazione il 15 marzo 1978.

(**) Lavoro eseguito nell'ambito della mia attività di ricerca come borsista C.N.R., presso I'Università di Genova. 
Per ogni $(t, x) \in A$ sia $Q(t, x)$ un sottoinsieme dello spazio $\boldsymbol{R}^{p+1}, v \geqslant 1$. Si dice che gli insiemi $Q(t, x)$ verificano la proprietà di Cesari in $A$, se per ogni $\left(t_{0}, x_{0}\right) \in A$ si ha:

$$
Q\left(t_{0}, x_{0}\right)=\bigcap_{\delta>0} \overline{\mathrm{co}^{0}} \bigcup_{\left|x-x_{0}\right|<\delta} Q\left(t_{0}, x\right) .
$$

Una funzione reale $(y, z) \mapsto f(y, z)$ è di Carathéodory in $B \times C$ se $f(y, \cdot)$ è continua in $O$ per q.o. $y \in B$ e se $f(\cdot, z)$ è misurabile in $B$ per ogni $z \in C$.

$L^{p}$ è 1'usuale spazio di Lebesgue $(1 \leqslant p \leqslant \infty)$ a valori vettoriali individuati dal contesto. Se $1<p<\infty, \dot{p}$ ne è il coningato di Lebesgue. Le successioni estratte sono indicate con la stessa notazione della successione originale.

Un operatore di Urysohn $w \mapsto \int_{\Omega} A(\cdot, s, w(s)) d s$, (essendo mis $(\Omega)<\infty$ ), si dice « regolare in $L^{p} "(1 \leqslant p \leqslant \infty)$ se applica $L^{p}$ in sè e se $\int_{\Omega}|A(\cdot, s, w(\cdot, s))| d s \in L^{p}$ per ogni $w$ misurabile in $\Omega^{2}$ tale che $|w(t, s)| \leqslant w_{0}(s)$ q.o. in $\Omega^{2}$, con $w_{0} \in L^{p}$ (v. [5]).

Defintzione. - $(x, u)$ è una coppia ammissibile se: $x: \Omega \mapsto \boldsymbol{R}^{n}$ sta in $L^{p}, u: \Omega \mapsto \boldsymbol{R}^{m}$ è misurabile e valgono:

(i) $x(t) \in A(t)$ q.o. in $\Omega$.

(ii) $x(t)=\varphi(t)+\int_{\Omega}[h(t, s, x(s))+g(t, s, x(s)) f(s, x(s), u(s))] d s$ q.o. in $\Omega$.

(iii) $u(t) \in U(t, x(t))$ q.o. in $\Omega$.

(iv) $f_{0}(\cdot, x(\cdot), u(\cdot)) \in L^{1}(\Omega)$.

Sia $\mathcal{A}$ l'insieme delle coppie ammissibili. Il problema è determinare una coppia $(x, u) \in \mathfrak{A}$ che minimizzi il funzionale:

$$
I(x, u)=\int_{\Omega} f_{0}(t, x(t), u(t)) d t
$$

\section{IPOTESI:}

(1) Per ogni $t \in \Omega, A(t)$ è chiuso; $M$ è chiuso.

(2) $\varphi \in L^{p}, h, g$ sono di Carathéodory in $\Omega^{2} \times \boldsymbol{R}^{n} ; f$ è di Carathéodory in $\Omega \times\left[\boldsymbol{R}^{n} \times \boldsymbol{R}^{m}\right] ; f_{0}$ ̀̀ integranda normale in $\Omega \times\left[\boldsymbol{R}^{n} \times \boldsymbol{R}^{m}\right]$ (v. [4]).

(3) $f_{0}(t, x, u) \geqslant f_{1}(t, x)$ q.o. $t \in \Omega, x \in A(t), u \in U(t, x)$, con

$$
\lim _{\|x\|_{L^{p} \rightarrow \infty}} \int_{\Omega} f_{1}(t, \infty(t)) d t=+\infty \quad x(t) \in A(t) \text { q.o. in } \Omega
$$

(4) $x \mapsto \int_{\Omega} h(\cdot, s, x(s)) d s$ è un operatore di Urysohn regolare da $L^{p}$ in $L^{p}$ e

$$
\underset{\operatorname{mis} D \rightarrow 0}{\lim \sup }\left\{\left\|\int_{D} h(\cdot, s, x(s)) d s\right\|_{L^{m}}:\|x\|_{L^{p} \leqslant 1}\right\}=0 .
$$


Marta Lutsa Bennati: Un teorema di esistenza per controlli ottimi, ece.

(5) $|g(t, s, x)| \leqslant \alpha(t)$ con $\alpha \in L^{p}$ per q.o. $t \in \Omega$, q.o. $s \in \Omega, \forall x \in \boldsymbol{R}^{n}$.

(6) $|f(t, x, u)|^{p} \leqslant a(t)+b f_{0}(t, x, u)$, ove $a \in L^{1}, b>0$ q.o. $t \in \Omega, x \in A(t), u \in U(t, x)$.

(7) $Q(t, x)=\left\{(\eta, \xi): \eta \geqslant f_{0}(t, x, u), \xi=f(t, x, u), u \in U(t, x)\right\}$ ha la proprietà di Cesari in $A$.

Sono date condizioni sufficienti per l'esistenza di controlli ottimi per il problema di ottimizzazione enunciato e si ha il seguente:

Teorfma. - Se sono verificate le ipotesi da (1) a (7), se $1<p<\infty$ e se la classe $\mathcal{A}$ delle coppie ammissibili è non vuota, allora esiste una coppia ottimale.

DIMOSTRAZIONE:

$1^{\circ}$ passo: esistenza di una successione minimizzante $\left\{\left(x_{k}, u_{k}\right)\right\}_{k \in N}$ in $\mathcal{A}$ tale che

$$
\left\{x_{k}\right\}_{k \in N} \text { è relativamente compatto in } L^{p} \text {. }
$$

Sia $\mu=\inf \{I(x, u):(x, u) \in \mathcal{A}\}$. Poiché $\mathcal{A}$ è non vuoto per ipotesi e per la (6) $f_{0}$ è inferiormente limitata da funzione sommabile, $\mu$ è reale. Esiste, dunque, una successione $\left(x_{k}, u_{k}\right) \in \mathcal{A}$ minimizzante per cui:

$$
\begin{aligned}
& (8) I\left(x_{k}, u_{k}\right)=\int_{\Omega} t_{0}\left(t, x_{k}(t), u_{k}(t)\right) d t \underset{k \rightarrow \infty}{\longrightarrow} \mu, \mathrm{e} \\
& \text { (9) } x_{k}(t)=\varphi(t)+\int_{\Omega}\left[h\left(t, s, x_{k}(s)\right)+g\left(t, s, x_{k}(s)\right) f\left(s, x_{k}(s), u_{k}(s)\right)\right] d s \text { q.o. in } \Omega \text {. Dalla }
\end{aligned}
$$

(3) segue che $\sup _{k}\left\|x_{k}\right\|_{L} p<+\infty$.

Dalla (4) segue che è applicabile il teorema 4, pp. 541 di $[5]$, per cui $\int_{\Omega} h\left(\cdot, s, x_{k}(s)\right) d s$ è relativamente compatto in $L^{p}$.

Analizziamo $t \mapsto \int_{\Omega} g\left(t, s, x_{k}(s)\right) f\left(s, x_{k}(s), u_{k}(s)\right) d s . \Delta$ tale scopo conviene definire il seguente operatore đi Urysohn: se $x \in L^{p}$ e $y \in L^{\dot{p}}, q=\min (p, \dot{p})$, consideriamo:

$$
(x, y) \mapsto \int_{\Omega} g(\cdot, s, x(s)) y(s) d s, \quad \text { da } L^{q} \text { in } L^{\nu}
$$

L'ipotesi (5) ci assicura che (10) è regolare da $L^{q}$ in $L^{p}$ e tale che

$$
\lim _{\operatorname{mis} D \rightarrow 0 \|} \sup _{D \rightarrow(x, v) \|_{L^{Q} \leqslant 1}}\left\|\int_{D} g(t, s, x(s)) y(s) d s\right\|_{L^{p}}=0 .
$$

Proviamo questa affermazione. Ovviamente (10) ha valori in $L^{p}$.

Per ogni coppia di funzioni misurabili in $\Omega^{2},(v(t, s), w(t, s))$ tali che

$$
|(v(t, s), w(t, s))|=|v(t, s)|+|w(t, s)| \leqslant v_{0}(s), \quad \text { con } v_{0} \in L^{q},
$$

13 - Annali di Matematica 
si ha:

$$
\int_{\Omega}|g(t, s, v(t, s)) w(t, s)| d s \in L^{p}
$$

Infatti:

$$
\begin{aligned}
\int_{\Omega}\left(\int_{\Omega}|g(t, s, v(t, s)) w(t, s)| d s\right)^{p} d t \leqslant & \int\left(\int_{\Omega} \alpha(t) v_{0}(s) d s\right)^{p} d t= \\
& \left.=\int_{\Omega} \alpha^{p}(t) d t\left(\int_{\Omega} v_{0}(s) d s\right)^{p} \leqslant\|\alpha\|_{L^{p}}^{p} \text { mis } \Omega\right)^{p / q}\left\|v_{0}\right\|_{L^{q}}^{p}<+\infty
\end{aligned}
$$

e ciò prova la regolarità. Inoltre

$$
\begin{aligned}
& \int_{\Omega}\left|\int_{D} g(t, s, x(s)) y(s) d s\right|^{p} d t \leqslant \iint_{D}\left(\int_{D} \alpha(t)|y(s)| d s\right)^{p} d t= \\
& =\int_{\Omega} \alpha^{p}(t) d t\left(\int_{D}|y(s)| d s\right)^{p} \leqslant\left\|\left.\alpha\right|_{L^{p}} ^{p}(\operatorname{mis} D)^{p / q}\left(\int_{D}|y(s)|^{\alpha} d s\right)^{p / q} \leqslant\right\| \alpha\left\|_{L^{p}}^{p}(\operatorname{mis} D)^{p / \dot{\alpha}}\right\| y \|_{L^{q}}^{p} .
\end{aligned}
$$

Si ottiene che (11) è vera. Per il teorema 4 di [5], (10) è completamente continuo da $L^{q}$ in $L^{p}$. Essendo $x_{k s}$ limitata in $L^{p}$ e $y_{k}(s)=f\left(s, x_{k}(s), u_{k}(s)\right)$ limitata in $L^{\dot{p}}$ per la (6) e per la (8), $\left(x_{k}, y_{k}\right)$ è limitata in $L^{q}$; ne segue che $t \rightarrow \int_{\Omega} g\left(t, s, x_{k}(s)\right)$ $f\left(s, x_{k}(s), u_{l e}(s)\right) d s$ è relativamente compatto in $L^{p}$.

E provato che $\left\{x_{k}\right\}_{k \in N}$ è relativamente compatto in $L^{p}$ e l'esistenza di una funzione $x$ in $L^{p}$ tale che per un'estratta $x_{k} \rightarrow \infty$ in $L^{p}$ e per un'ulteriore estratta $x_{k}(t) \rightarrow x(t)$ q.o. in $\Omega$.

Per la (i) $x_{k t}(t) \in A(t)$ q.o. in $\Omega$ e per la (1) $x(t) \in A(t)$ q.o. in $\Omega$. La funzione $x$ verifica (i). Per la (6) e la (8) $\sup _{k}\left\|y_{k}\right\|_{L^{\dot{j}}}<+\infty$. Per un'estratta $y_{k} \rightarrow y$ in $L^{\dot{p}}$.

$2^{\circ}$ passo: esistenza di una funzione $\lambda$ integrabile in $\Omega$ tale che

$$
(\lambda(t), y(t)) \in Q(t, x(t)) \text { q.o. in } \Omega \text { e } \int_{\Omega} \lambda(t) d t \leqslant \mu .
$$

Da $y_{k} \rightarrow y$ in $L^{\dot{p}}$, per il teorema di Mazur, esiste una successione di combinazioni convesse di $y_{k}$ che converge a $y$ fortemente in $L^{\dot{p}}$.

Per ogni intero $j$, ci sono un interno $n_{j}$ e un insieme di interi $i=1,2, \ldots, r=r(j)$, un insieme di numeri $a_{1 j}, \ldots, a_{r j}$, tali che

e se

$$
a_{i j} \geqslant 0, \quad i=1,2, \ldots, r, \sum_{i=1}^{r} a_{i j}=1, n_{i+1}>n_{i}+r(j)
$$

$s_{i}=\sum_{i=1}^{r} a_{i j} y_{n_{j}+i}$, allora $s_{j} \rightarrow y$ in $L^{\dot{p}}$. Per un'estratta

$$
s_{i}(t) \rightarrow y(t) \quad \text { q.o. in } \Omega .
$$


Corrispondentemente a questa successione definiamo con gli stessi $a_{i j}$ e indici $n_{j}$ la successione:

$$
\lambda_{j}(t)=\sum_{i=1}^{r} a_{i j} f_{0}\left(t, x_{n_{j}+i}(t), u_{n_{j}+i}(t)\right) \quad \text { in } \Omega
$$

e

$$
\lambda(t)=\min _{j} \lim \lambda_{j}(t), \quad t \in Q .
$$

Per la (6) $\lambda$ è inferiormente limitata da funzione sommabile.

Inoltre applicando il lemma di Fatou si ha:

$$
\begin{aligned}
\int_{\Omega} \lambda(t) d t=\int_{\Omega} \min \lim _{j} \lambda_{j}(t) d t \leqslant \min \lim \int_{\Omega} & \lambda_{j}(t) d t= \\
& =\min \lim _{j} \int_{\Omega} \sum_{i=1}^{r} a_{i j} f_{0}\left(t, x_{n_{j}+i}(t), u_{n_{j}+i}(t)\right) d t= \\
& =\min \lim _{i=1} \sum_{i=1}^{r} a_{i j} \int_{\Omega} f_{0}\left(t, x_{n_{j}+i}(t), u_{n_{j}+i}(t)\right) d t=\mu,
\end{aligned}
$$

per il teorema di Cesaro sul limite di una successione di combinazioni convesse, dato che

$$
\int_{\Omega} f_{0}\left(t, x_{n_{j}+i}(t), u_{n_{j}+i}(t)\right) d t=I\left(x_{n_{j}+i}, u_{n_{j}+i}\right) \underset{j \rightarrow \infty}{\longrightarrow} \mu .
$$

$\lambda(t)$ è finito q.o. in $\Omega$, perché $\lambda \in L^{1}(\Omega)$, in quanto $\int_{\Omega} \lambda(t) d t \leqslant \mu$. Inoltre $\left(x_{k}, u_{k}\right) \in \mathcal{A}$, per eui:

$$
\left(f_{0}\left(t, x_{n_{j}+i}(t), u_{n_{j}+i}(t)\right), f\left(t, x_{n_{j}+i}(t), u_{n_{j}+i}(t)\right)\right) \in Q\left(t, x_{n_{j}+i}(t)\right)
$$

per q.o. $t \in \Omega$. 亡̀ stato provato che:

$$
x_{n,+i}(t) \underset{j \rightarrow \infty}{\longrightarrow} x(t) \quad \text { q.o. in } \Omega .
$$

Fissiamo $t$ per cui sono verificate (12), (13), (14) e (15).

Per ogni $\delta>0$ esiste $j_{0}$ (dipendente da $\delta$ e $t$ ) tale che se $j>j_{0}, i=1,2, \ldots, r(j)$, $\left|x_{n,+i}(t)-x(t)\right|<\delta$, da cui

$$
\left(f_{0}\left(t, x_{n_{j}+i}(t), u_{n_{j}+i}(t)\right), f\left(t, x_{n_{j}+i}(t), u_{n_{j}+i}(t)\right)\right) \in \bigcup_{[x-x(t)]<\delta} Q(t, x)
$$

se $j>j_{0}, i=1,2, \ldots, r(j)$ e $\left(\lambda_{j}(i), s_{j}(i)\right) \in \mathrm{co} \bigcup Q(t, x), j>j_{0}$.

Passando al limite su $j,(\lambda(t), y(t)) \in \overline{\mathrm{co}} \bigcup_{|x-\infty(t)|<\delta}^{|x-x(t)|<\delta} Q(t, x)$. 
Per l'arbitrarietà di $\delta(\lambda(t), y(t)) \in \bigcap_{\delta>0} \overline{c 0} \bigcup_{|x-x(t)|<\delta} Q(t, x)$ e per la $(7)(\lambda(t), y(t)) \in Q(t, x(t))$ e ciò vale q.o. in $\Omega$ per l'arbitrarietà di $t$.

Per la definizione dell'insieme $Q(t, x(t))$ risulta: $\lambda(t) \geqslant f_{0}(t, x(t), u), y(t)=f(t, x(t), u)$ per qualche $u \in U(t, x(t))$ q.o. in $\Omega$.

$3^{\circ}$ passo: esistenza di una funzione $u$ misurabile in $\Omega$ tale che:

$$
u(t) \in U(t, x(t)), y(t)=f(t, x(t), u(t)), \lambda(t) \geqslant f_{0}(t, x(t), u(t)), \text { q.o. in } \Omega .
$$

Si sfrutta un teorema di selezione misurabile. Per ogni $t \in \Omega$ la multiapplicazione $U(t, \cdot)$ che ad ogni $x \in A(t)$ associa l'insieme $U(t, x)$, ha grafico chiuso, ove

$$
\operatorname{gph} U(t, \cdot)=\left\{(x, y) \in \boldsymbol{R}^{n} \times \boldsymbol{R}^{m}: x \in A(t), y \in U(t, x)\right\} .
$$

Infatti se $\left(x_{k}, y_{k}\right) \in \operatorname{gph} U(t, \cdot), x_{k} \rightarrow x, y_{k} \rightarrow y, x_{k} \in A(t), y_{k} \in U\left(t, x_{k}\right)$, per la (1) si ha che $x \in A(t)$ e $y \in U(t, x)$ e quindi $(x, y) \in \operatorname{gph} U(t, \cdot)$.

Inoltre la multiapplicazione $G$ ehe ad ogni $t \in \Omega$ associa $g p h U(t, \cdot)$ ha valori chiusi ed è misurabile. Infatti,

$$
\begin{aligned}
\operatorname{gph} G=\left\{(t, w) \in \Omega \times\left[\boldsymbol{R}^{n} \times \boldsymbol{R}^{m}\right]: t \in \Omega, w \in \operatorname{gph} U(t, \cdot)\right\}= \\
\quad=\{(t, x, y): t \in \Omega, x \in A(t), y \in U(t, x)\}=M
\end{aligned}
$$

è chiuso, per cui, per il teorema 1E pp. 164 di $[6], G$ è misurabile. $x(t)$ (la funzione ottenuta come limite della successione minimizzante) è misurabile.

Allora per il teorema $1 \mathrm{~N}$ pp. 171 di [6], la multiapplicazione $U(t, x(t))$ è misurabile (e a valori chiusi). Usando il teorema $2 \mathrm{~J}$ pp. 178 di [6] la multiapplicazione:

$$
\Gamma(t)=\left\{u \in U(t, x(t)): \lambda(t) \geqslant f_{0}(t, x(t), u), y(t)=f(t, x(t), u)\right\}
$$

ammette selezioni misurabili, e quindi esiste $u: \Omega \rightarrow \boldsymbol{R}^{m}$ misurabile tale che per q.o. $t \in \Omega, u(t) \in U(t, x(t)), \lambda(t) \geqslant f_{0}(t, x(t), u(t)), y(t)=f(t, x(t), u(t))$, e cio conclude il $3^{\circ}$ passo.

La funzione misurabile $u$ verifica (iii). Poiché $f_{0}$ è inferiormente limitata da funzione sommabile per la $(6)$ e $f_{0}(t, x(t), u(t)) \leqslant \lambda(t)$ q.o. in $\Omega$, con $\int_{\Omega} \lambda(t) d t \leqslant \mu$, risulta $f_{0}(\cdot, x(\cdot), u(\cdot)) \in L^{\mathbf{1}}$; ̀̀ verificata (iv) e

$$
\int_{\Omega} f_{0}(t, x(t), u(t)) d t \leqslant \mu=\inf \{I(x, u):(x, u) \in \mathcal{A}\} .
$$

$4^{\circ}$ passo: esistenza di una coppia $(x, u)$ ottimale.

Se si verifica che vale (ii), allora $(x, u)$ è coppia ammissibile e poiché vale (16), $(x, u)$ è ottimale.

$$
x_{k}(t) \rightarrow x(t) \quad \text { q.o. in } \Omega .
$$


$\int_{\Omega} h(\cdot, s, x(s)) d s$ è un operatore di Urysohn regolare da $L^{p}$ in $L^{p}$ per l'ipotesi (4) e per il teorema 3 , pp. 541 di [5] è continuo da $L^{p}$ in $L^{p}$, per cui, avendosi $x_{k} \rightarrow x$ in $L^{p}$, risulta:

$$
\int_{\Omega} h\left(\cdot, s, x_{k}(s)\right) d s \rightarrow \int_{\Omega} h(\cdot, s, x(s)) d s
$$

in $L^{p}$ e per un'estratta

$$
\int_{\Omega} h\left(t, s, x_{l l}(s)\right) d s \rightarrow \int_{\Omega} h(t, s, x(s)) d s \quad \text { q.o. in } \Omega .
$$

Per la (5) q.o. in $\Omega$ l'operatore di Niemytskij $x \rightarrow g(t, \cdot, x(\cdot))$ è continuo da $L^{p}$. in $L^{p}$ (v. [7]), e pertanto $g\left(t, \cdot, x_{k}(\cdot)\right) \rightarrow g(t, \cdot, x(\cdot))$ in $L^{p}$ per q.o. $t \in \Omega$. Poiché $f\left(\cdot, x_{k}(\cdot), u_{k}(\cdot)\right) \rightarrow f(\cdot, x(\cdot), u(\cdot))$ in $L^{\dot{p}}$, per q.o. $t \in \Omega$ si ha:

$$
\int_{\Omega} g\left(t, s, x_{k}(s)\right) f\left(s, x_{k}(s), u_{k}(s)\right) d s \rightarrow \int_{\Omega} g(t, s, x(s)) f(s, x(s), u(s)) d s .
$$

Usando (17), (18), (19), e passando al limite su $k$ in (9), si ottiene che $(x, u)$ verifica (ii) È così completamente dimostrato il teorema di esistenza enunciato.

EsEmpio. - Sia $r=n=m=1 . \Omega=[0,1]$.

Si vuole minimizzare il funzionale:

$$
I(x, u)=\int_{0}^{1}(x(t))^{2}+(u(t))^{2} d t
$$

ove $x(t)$ soddisfa la seguente equazione integrale:

$$
x(t)=\frac{1}{\sqrt[3]{t}}+\int_{0}^{1}\left\{\frac{\operatorname{sen}\left(s^{2}\right) \operatorname{sen}\left(t^{2}\right)}{3+\operatorname{arctg} x(s)}+\frac{\cos (s x(s))}{\sqrt[3]{1-t}}|u(s)|\right\} d s,
$$

con $x \in L^{2},(p=\dot{p}=2), x(t) \in A(t)$ q.o. in $[0,1]$, ove per ogni $t \in(0,1]$

$$
\begin{aligned}
& A(t)=\left[-1-\frac{1}{t}, 1+\frac{1}{t}\right], \quad A(0)=\boldsymbol{R} . \\
& A=\{(t, x) \in[0,1] \times \boldsymbol{R}: t \in[0,1], x \in A(t)\} .
\end{aligned}
$$

Per ogni $(t, x) \in A$ sia

$$
U(t, x)=\{u \in \boldsymbol{R}:|u| \leqslant t+|x|\}, \quad M=\{(t, x, u):(t, x) \in A, u \in U(t, x)\}
$$

Per q.o. $t \in[0,1], u(t) \in U(t, x(t))$. 
Per potere applicare il teorema di esistenza devono essere verificate le ipotesi da (1) a (7) e devono esistere delle coppie ammissibili. $A(t)$ ha valori chiusi in $[0,1]$ e $M$ è chiuso.

$\varphi(t)=1 / \sqrt[3]{t}$ è una funzione in $L^{2} . f_{0}(t, x, u)=x^{2}+u^{2}$ è continua in $[0,1] \times \boldsymbol{R} \times \boldsymbol{R}$ e verifica $(3)$ con $f_{1}(t, x)=x^{2}$.

$$
h(t, s, x)=\frac{\operatorname{sen}\left(s^{2}\right) \operatorname{sen}\left(t^{2}\right)}{3+\operatorname{arctg} x} \quad \text { è continua in }[0,1] \times[0,1] \times \boldsymbol{R} .
$$

Poiché $|h(t, s, x)| \leqslant 1$ in $[0,1] \times[0,1] \times \boldsymbol{R}$, si verifica facilmente che $x \rightarrow \int_{0}^{1} h(\cdot, s, x(s)) d s$ è un operatore di Uxysohn regolare da $L^{2}$ in $L^{2}$ e inoltre

$$
\lim _{\operatorname{mis}} \sup _{D \rightarrow 0}\left\{\left\|\int_{D} h(\cdot, s, x(s)) d s\right\|_{L^{2}}:\|x\|_{L^{2}} \leqslant 1\right\}=0,
$$

per cui $h$ verifica (4).

$g(t, s, x)=\cos (s x) / \sqrt[3]{1-t}$, è funzione di Carathéodory in $[0,1]^{2} \times \boldsymbol{R}$, e $|g(t, s, x)| \leqslant$ $\leqslant 1 / \sqrt[3]{1-t}$ q.o. $t \in[0,1], \forall s \in[0,1], x \in \boldsymbol{R}$, e vale la $(5)$ con $\alpha(t)=1 / \sqrt[3]{1-t} \in L^{2}$.

$f(t, x, u)=|u|$ è continua in $[0,1] \times \boldsymbol{R} \times \boldsymbol{R}$.

$|f(t, x, u)|^{2}=|u|^{2} \leqslant x^{2}+u^{2}$ per ogni $t \in[0,1], x \in A(t), u \in U(t, x)$ e vale $(6)$ con $a(t)=0, b=1$.

Per ogni $(t, x) \in A, Q(t, x)=\left\{(\eta, \xi): \eta \geqslant x^{2}+u^{2}, \xi=|u|,|u| \leqslant t+|x|\right\}$ è un sottoinsieme convesso e chiuso di $\boldsymbol{R}^{2}$ e si vede facilmente che $Q(t, x)$ ha la proprietà di Cesari in $A$.

Sono perciò verificate le ipotesi da (1) a (7) del teorema di esistenza. La classe $A$ delle coppie ammissibili è non vuota.

Scegliendo $u(t)=0$, l'equazione di Hammerstein:

$$
x(t)=\frac{1}{\sqrt[3]{t}}+\int_{0}^{1} \frac{\operatorname{sen}\left(s^{2}\right) \operatorname{sen}\left(t^{2}\right)}{3+\operatorname{arctg} x(s)} d s
$$

ha una sola soluzione $x(t)$ per il teorema 1, pp. 126 di [8].

Inoltre

$$
|x(t)| \leqslant \frac{1}{\sqrt[3]{t}}+1 \quad \text { in }(0,1], \quad \text { e } \quad x(t) \in A(t) \quad \text { in }[0,1]
$$

$u(t) \in \delta(t, x(t)) \quad$ in $[0,1], \quad$ e $\quad(x(t))^{2}+(u(t))^{2}=(x(t))^{2} \leqslant \frac{1}{\sqrt[3]{t^{2}}}+1+\frac{2}{\sqrt[3]{t}} \in L^{1}$

La coppia $(x, u)$, verificando (i), (ii), (iii), (iv), è ammissibile.

Esiste, dunque, una coppia ottimale per il problema dato. 
NOTE:

(a) Nel caso in cui la funzione $g$ è indipendente da $x$ e quindi l'equazione integrale di stato è del tipo:

$$
x(t)=\varphi(t)+\int_{\Omega}[h(t, s, x(s))+g(t, s) f(s, x(s), u(s))] d s
$$

si ha un teorema di esistenza con le stesse ipotesi da (1) a (7) eccetto la (5) che viene modificata con l'ipotesi che $g \in L^{P}(\Omega \times \Omega)$. Nel caso in cui $h=0$ si ha un'equazione integrale del tipo di Hammerstein.

(b) Il teorema di esistenza provato include il caso differenziale ove

$$
\varphi(t)=x_{0}, \quad g(t, s)= \begin{cases}1, & t_{0} \leqslant s \leqslant t, \\ 0, & t<s \leqslant t_{1}\end{cases}
$$

essendo l'equazione di stato $\dot{x}=f(t, x, u)$ in $\left[t_{0}, t_{1}\right], x\left(t_{0}\right)=x_{0}$, ossia

$$
x(t)=x_{0}+\int_{t_{\mathrm{a}}}^{t} f(s, x(s), u(s)) d s \quad \text { in }\left[t_{0}, t_{1}\right] .
$$

Ovviamente $g$ verifica (5).

(c) Il teorema di esistenza provato è in condizioni più generali di alcuni risultati noti sull'argomento ( $\mathrm{v} .[1],[2])$. In [1] sono dimostrati due teoremi concernenti l'esistenza di controlli ottimi per sistemi di equazioni di Urysohn e problemi di Lagrange che si deducono come casi particolari del teorema precedente.

In un primo teorema (teorema 1-2, pp. 103 di [1]), infatti si ha un'equazione della forma

$$
x(t)=\int_{\Omega}[A(t, s, x(s))+B(t, s)] u(s) d s
$$

con $x \in L^{p} \quad(1 \leqslant p \leqslant \infty)$ \& $u \in L^{q}(1<q<\infty)$, e $\|u\|_{L} q \leqslant N \quad(N>0)$.

In questo caso risulta $f(t, x, u)=u, h=0$ e $g(t, s, x)=A(t, s, x)+B(t, s)$; sono date condizioni sufficienti affinchè l'operatore

$$
(x, u) \mapsto \int_{\Omega}[A(\cdot, s, x(s))+B(\cdot, s)] u(s) d s
$$

trasformi parti limitate di $L^{1} \times L^{q}$ in parti relativamente compatte di $L^{1}$. Nel secondo teorema (teorema 2-2, pp. 106 di [1]), si ha il seguente sistema di equazioni integrali:

$$
x(t)=\int_{\Omega}\left[A(t, s, x(s))+B_{1}(t, s) B_{2}(s, u(s))\right] d s,
$$

con $x \in L^{p}(1 \leqslant p<\infty), u \in L^{q}(1 \leqslant q \leqslant \infty)$ e $\|u\|_{L} q \leqslant N$. 
$A(t, s, x)=h(t, s, x)$ e si ha l'indipendenza dallo stato $x$ dei termini $g(t, s, x)=$ $=B_{1}(t, s), f(t, x, u)=B_{2}(t, u)$.

$x \mapsto \int_{\Omega} A(\cdot, s, x(s)) d s$ si suppone sempre essere un operatore di Urysohn regolare in $L^{p}$, con la condizione (4) del teorema qui provato che lo rende completamente continuo. Si suppone che esista $s>1$ t.c.

$$
\sup \left\{\int_{\Omega}\left|B_{2}(s, u(s))\right|^{s} d s: u \in \mathcal{U} \text { classe dei controlli ammissibili }\right\}<+\infty
$$

e

$$
\int_{\Omega}\left(\int_{\Omega}\left|B_{1}(t, s)\right|^{\dot{s}} d s\right)^{p / s} d t<+\infty
$$

L'ipotesi (6) del teorema dimostrato assicura che la condizione su $B_{2}$ è vera con $s=\dot{p}$ ed è sufficiente sui sottoinsiemi di $A$ per cui $I(x, u)<+\infty$ e la condizione su $B_{1}$ è ancora $B_{1} \in L^{p}(\Omega \times \Omega)$. Si conclude che questo lavoro è una estensione dei risultati di [1].

In [2] è dato un teorema di esistenza per il controllo ottimo di equazioni integrali non lineari di Volterra del tipo:

$$
x(t)=x\left(t_{1}\right)+\int_{i_{1}}^{t}\left[h_{1}(t, s, x(s))+g_{1}(t, s, x(s)) f_{1}(t, s, u(s))\right] d s, \quad t_{1} \leqslant t \leqslant t_{2} .
$$

$x:\left[t_{1}, t_{2}\right] \rightarrow \boldsymbol{R}^{n}$ è continua, $u:\left[t_{1}, t_{2}\right] \rightarrow \boldsymbol{R}^{m}$ è misurabile e ci sono vincoli del tipo: $(t, x(t)) \in A \subset \boldsymbol{R}^{n+1}$ in $\left[t_{1}, t_{2}\right]$ e $u(t) \in U(t, x(t))$ q.o. in $\left[t_{1}, t_{2}\right]$ con $U(t, x) \subset \boldsymbol{R}^{m}$ per ogni $(t, x) \in A$.

Risulta evidentemente:

$$
\begin{aligned}
& h(t, s, x)= \begin{cases}h_{1}(t, s, x), & t_{1} \leqslant s \leqslant t \\
0, & t<s \leqslant t_{2},\end{cases} \\
& g(t, s, x)= \begin{cases}g_{1}(t, s, x), & t_{1} \leqslant s \leqslant t \\
0, & t<s \leqslant t_{2},\end{cases} \\
& f(t, x, u)=f_{1}(t, x, u),
\end{aligned}
$$

Si vuole sempre minimizzare un funzionale della forma:

$$
I(x, u)=\int_{t_{1}}^{t_{2}} f_{0}(t, x(t), u(t)) d t .
$$

Le ipotesi di compattezza su $A$ e di continuità su $h_{1}$ e $g_{1}$ fanno si che ovviamente $h$ e $g$ verificano (4) e (5) del presente teorema. Tali condizioni sono verificate anche 
quando più generalmente $A$ è chiuso e si fanno ulteriori ipotesi di limitatezza sulla funzione $g_{1}$ e si impone che esista una funzione $w$ monotona non decrescente, $w(0)=0$ tale che per ogni $t, t^{\prime}\left|h_{1}(t, s, x)-h_{1}\left(t^{\prime}, s, x\right)\right| \leqslant w\left(\left|t-t^{\prime}\right|\right)$ e per qualche $t^{*}\left|h_{1}\left(t^{*}, s, x\right)\right| \leqslant$ $\leqslant \psi(s)$ con $\psi \in L^{\mathbf{1}}$.

Le funzioni $f_{1}, f_{0}$ soddisfano la seguente condizione di crescita: per ogni $\varepsilon>0$ esiste una funzione $\psi_{\varepsilon}$ localmente integrabile, $\psi_{\varepsilon} \geqslant 0$ tale che ${ }_{\varepsilon}^{*}\left|f f_{1}(t, x, u)\right| \leqslant \psi_{\varepsilon}(t)+$ $+\varepsilon f_{0}(t, x, u)$ in $M$.

Il risultato di [2] è quindi ancora un caso particolare del presente lavoro. In [3] è provato un teorema di esistenza per problemi di Lagrange in cui l'equazione di stato è un'equazione integrale astratta:

$$
(\mathfrak{L} x)(t)=\phi\left(t,(\mathcal{H} x)(t), \int_{\Omega} k(t, s,(\mathcal{K}(x)(s)) f(s,(\mathcal{H} x)(s), u(s)) d s)\right.
$$

con $x \in S$ spazio topologico, $\mathcal{L}, \mathcal{H}$ operatori da $\mathbb{S}$ in $L^{1},(\mathcal{H} x)(t) \in A(t)$ e $u(t) \in$ $\in U(t,(\mathcal{H} x)(t))$ q.o. in $\Omega$. Per ogni $t \in \Omega, A(t) \subset \boldsymbol{R}^{n}$, e se $t \in \Omega, x \in A(t), U(t, x) \subset \boldsymbol{R}^{m}$. Si vuole minimizzare

$$
I(x, u)=\int_{\Omega} f_{0}(t,(\mathcal{M} x)(t), u(t)) d t
$$

$\phi$ è una funzione continua. L'equazione di Urysohn del presente teorema è un caso concreto in cui $(\mathcal{L} x)(t)=x(t),(\mathscr{L} x)(t)=x(t)$ e il secondo membro di $(20)$ è funzione della terza variabile, per cui $h=0, g(t, s, x)=k(t, s, x)$. In [3] si assume l'insieme delle traiettorie ammissibili relativamente compatto in $L^{p}$, mentre nel teorema presente sono date condizioni sufficienti affinché ciò avrenga.

\section{BIBLIOGRAFIA}

[1] T. Zowezzr, Esistenza e condizioni necessarie per controlli ottimi di sistemi definiti da equazioni integrali, Annali di Matematica pura ed applicata, (IV), 91 (1972), pp. 97-118.

[2] T. S. ANGELL, On the optimal control of systems governed by nonlinear Volterra equations, J.O.T.A., 19 (1976), pp. $29-45$.

[3] D. E. CowLes, An existence theorem for optimization problems involving integral equations, SIAM J. Control, 11 (1973), pp. 595-606.

[4] R. T. Rockafeliar, Inegrals which are convex functionals, Pacifie. J. Math., 24 (1968), pp. 525-539.

[5] P. P. ZABReiko, Continuity and complete continuity of Urysohn operators, Soviet. Math. Doklady, 6 (1965), pp. 540-544.

[6] R. T. ROCKAFELLAR, Integral functionals, normal integrand, and measurable selections, in Nonlinear Operators and the Calculus of Variations, Bruxelles, 1975.

[7] M. A. Krasnosex'sku, Topological methods in the theory of nonlinear integral equations, Pergamon Press, 1964.

[8] H. Brezrs - F. E. Browder, Nonlinear integral equations and systems of Hammerstein type, Advances in Mathematics, 18 (1975), pp. $115-147$. 